\title{
Commentaire
}

\section{L'environnement alimentaire et les populations vulnérables : défis et possibilités en matière de politiques}

\author{
Lana Vanderlee, Ph. D., rédactrice invitée (1); Dana Lee Olstad, Ph. D., R.D. (2)
}

Diffuser cet article sur Twitter

Le but de la politique sur l'environnement alimentaire est d'améliorer l'apport nutritionnel au niveau de la population afin d'améliorer la santé générale de cette dernière. On a cependant peu examiné jusqu'à présent la question des impacts différentiels possibles des effets des politiques et des interventions en matière d'environnement alimentaire sur les divers segments de la population.

Le niveau socioéconomique détermine les expositions et la vulnérabilité des individus aux conditions environnementales positives comme négatives. Étant donné la forte influence de l'environnement alimentaire sur l'apport nutritionnel et l'incidence de la nutrition sur la santé, on peut supposer que les inégalités en matière de santé soient attribuables au moins partiellement à une plus grande exposition ou à une plus grande vulnérabilité (ou les deux) aux effets causés par un environnement alimentaire malsain chez les groupes socioéconomiques défavorisés. Mieux comprendre les expositions différentielles et la vulnérabilité à un environnement alimentaire malsain au sein des populations à faible revenu (ou vulnérables d'un quelconque point de vue) conduit certainement à mieux intervenir pour aider les groupes défavorisés à atteindre leur plein potentiel en matière de santé. Cette édition spéciale du mois d'octobre réunit ainsi cinq articles traitant de la santé et des inégalités sociales en lien avec l'environnement alimentaire, dans le but de comprendre les interactions entre environnement alimentaire, politiques et équité en matière de santé.

Deux articles dans ce numéro spécial décrivent les défis en matière d'élaboration de politiques sur l'environnement alimentaire, ainsi que la nécessité, pour augmenter la sécurité alimentaire, de disposer d'une politique cohérente et globale tenant compte des nombreuses dimensions de l'environnement alimentaire.

L'article de Burnett et ses collègues souligne les problèmes récurrents liés à l'intervention du gouvernement fédéral en matière d'insécurité alimentaire dans le Nord canadien ${ }^{1}$. Les données indiquent que plus de la moitié des communautés étudiées n'avaient pas d'épicerie faisant compétition à la North West Company. La faible compétition dans le domaine de la vente au détail alimentaire constitue ainsi un facteur aggravant l'insécurité alimentaire dans les collectivités isolées. De plus, les réponses à un sondage mené auprès des membres de ces collectivités indiquent que la faible qualité, les prix plus élevés et la disponibilité restreinte des aliments périssables bons pour la santé peuvent inciter à l'achat d'aliments transformés et préemballés, souvent moins bénéfiques pour la santé. Les auteurs démontrent ainsi que l'échec des politiques à aborder d'une manière plus globale (au-delà des subventions alimentaires) les facteurs menant à la fois à ces prix trop élevés (en particulier la faible compétition dans le secteur de la vente au détail alimentaire) et à une qualité de nourriture inférieure a un lourd impact pour les populations des collectivités du Nord canadien.

L'article rédigé par Speed et ses collègues révèle un enjeu supplémentaire lié à l'élaboration d'une politique : inclure les interactions entre les différentes stratégies ou composantes de stratégies afin de réduire les conséquences négatives non prévues $^{2}$. Par exemple, une politique qui met de l'avant une augmentation de la consommation de produits frais peut mettre en danger la sécurité alimentaire en raison de la contamination microbienne. Cet article détaille les préoccupations d'un groupe d'intervenants en matière de salubrité alimentaire et de sécurité alimentaire communautaire en Colombie-Britannique et, surtout, il détermine un but commun : augmenter l'approvisionnement en aliments à la fois de haute qualité et sécuritaires afin d'aider à la création d'un environnement alimentaire plus sain pour les personnes souffrant d'insécurité alimentaire. Ainsi, une politique sur l'environnement alimentaire devant être globale et générale, le défi est de s'assurer que les stratégies tiennent aussi compte de la dynamique entre diverses composantes de l'environnement alimentaire, afin de maintenir la cohérence et de ne pas travailler à contrecourant.

Les trois articles suivants se concentrent principalement sur l'accessibilité physique à une alimentation saine dans l'environnement alimentaire de la vente au détail. Ils offrent de possibles solutions aux défis communément rencontrés. L'accessibilité géographique aux magasins d'aliments ayant une offre plus santé est l'un des aspects les plus étudiés de l'environnement alimentaire, surtout quant aux disparités de cette accessibilité entre secteurs à revenus élevés et secteurs à faibles revenus. Il est légitime de s'intéresser à l'environnement de la vente au détail dans la mesure où la majorité de la nourriture consommée par le grand public y est achetée ${ }^{3}$. La manière de distinguer magasins d'alimentation "plus santé » et " moins santé » demeure cependant un défi d'importance et, à la lumière de ce défi, se pose même la 
question de l'utilité de cette distinction pour la mise en œuvre des stratégies.

L'article rédigé par Minaker et ses collaborateurs se base sur des données de vente afin d'étudier les effets d'une intervention sur la vente au détail dans un dépanneur d'un quartier à faibles revenus de Toronto, méthode peu utilisée en recherche interventionnelle dans le commerce de détail ${ }^{4}$. Les dépanneurs ont tendance à offrir moins d'aliments sains, ce qui en fait une cible intéressante pour une intervention en environnement alimentaire, en particulier dans les quartiers à accessibilité restreinte à des épiceries traditionnelles de plus grande importance ${ }^{5}$. Cette étude préliminaire compare les données sur les tendances des ventes d'un dépanneur avant et après une intervention visant à augmenter sa disponibilité en fruits et légumes frais et fait naître des occasions pour les commerçants de tirer des bénéfices de la vente d'aliments plus sains. Étant donné que vendre des produits sains est considéré comme peu rentable, ce qui constitue un obstacle majeur à l'augmentation de la disponibilité de ces produits, intégrer des données sur les ventes dans les méthodologies de recherche pourrait lever cet obstacle au sein de l'industrie de la vente au détail alimentaire. Il est important de montrer qu'offrir des produits plus sains a un impact positif sur les profits, ce qui peut éviter de créer des stratégies, politiques ou interventions en ce domaine puisque les détaillants offriront naturellement les produits les plus rentables.

Slater et ses collègues étudient le concept de désert alimentaire et l'accessibilité à des épiceries de plus grande taille offrant une sélection plus variée de produits alimentaires sains pour les groupes de population à faibles revenus résidant à Winnipeg ${ }^{6}$. La présence d'une grande proportion de ménages à faibles revenus, en particulier dans le centre-ville, laisse présager le risque d'une santé médiocre pour nombre de résidents, et que ce risque soit potentiellement augmenté par une accessibilité moindre à des détaillants offrant des aliments plus sains. Les auteurs définissent les déserts alimentaires en utilisant des moyens relativement simples : ils utilisent des données facilement accessibles afin de simplifier le repérage de ces zones, fournissant ainsi une information destinée à étayer les décisions en matière de politiques visant une accessibilité plus équitable aux aliments sains.
Enfin, le rapport d'étape de Mahendra et ses collègues rend compte du projet de mise au point d'indicateurs universels destinés à décrire l'accessibilité à différentes sortes de commerces de détail en Ontario, avec un élargissement potentiel à d'autres provinces ou territoires voire à l'ensemble du Canada ${ }^{7}$. Au fur et à mesure que les politiques visant à améliorer l'environnement alimentaire vont évoluer, ces indicateurs vont pouvoir fournir un cadre de surveillance de leur incidence, en particulier pour les populations vulnérables sur le plan nutritionnel.

Les articles de ce numéro contribuent à une meilleure compréhension de l'environnement alimentaire actuel et de la façon dont il affecte les populations vulnérables, et à une meilleure compréhension également des difficultés à s'assurer que les politiques prennent en compte les multiples dimensions de l'environnement alimentaire pour que les répercussions sur la santé des populations soient équitables. Ila permettent également de dégager les opportunités d'avoir par ce biais une influence sur les facteurs sous-jacents aux inégalités alimentaires et en matière de santé, ces dernières coûtant au système de santé canadien 6,2 milliards de dollars par $a^{8}$. Enfin, ces articles nous rappellent l'importance de garder une optique d'équité sanitaire lors de la conception de politiques en matière d'environnement alimentaire à l'échelle de la population canadienne.

\section{Remerciements}

Lana Vanderlee est récipiendaire d'une bourse du Programme de bourses postdoctorales Banting des Instituts de recherche en santé du Canada.

\section{Références}

1. Burnett K, Skinner K, Hay T, LeBlanc J, Chambers L. Environnement alimentaire de la vente au détail et expériences de magasinage dans les collectivités des Premières nations du nord des provinces. Promotion de la santé et prévention des maladies chroniques au Canada. 2017;37(10):373-82.

2. Speed KA, Meyer SB, Hanning, RM, Majowicz SE. "Très transformés, très emballés et très mauvais pour la santé... mais à faible risque » : exploration des liens entre sécurité des aliments et salubrité des aliments dans les collectivités. Promotion de la santé et prévention des maladies chroniques au Canada. 2017;37(10):361-72.
3. Statistique Canada. Dépenses alimentaires moyennes des ménages, par province (Canada) [Internet]. Ottawa (Ont.) : Statistique Canada; 2014 [dernière modification 27 janv. 2017]. En ligne à : http://www.statcan.gc.ca /tables-tableaux/sum-som/102/cst01 /famil132a-fra.htm.

4. Minaker LM, Lynch M, Cook BE, Mah CL. Analyse de données sur les ventes lors d'une intervention axée sur un dépanneur santé de Toronto : le projet FRESH sur l'environnement de la vente d'aliments au détail comme déterminant de la santé. Promotion de la santé et prévention des maladies chroniques au Canada. 2017;37(10): 383-91.

5. Minaker LM, Shuh A, Olstad DL, Engler-Stringer R, Black JL, Mah CL. Recherche sur les environnements de vente au détail alimentaire au Canada : examen sur la portée. Can J Public Health. 2016;107:4-13.

6. Slater J, Epp-Koop S, Jakilazek M, Green C. Déserts alimentaires à Winnipeg (Canada) : une nouvelle méthodologie de mesure d'un concept complexe et controversé. Promotion de la santé et prévention des maladies chroniques au Canada. 2017;37(10): 392-9.

7. Mahendra A, Polsky JY, Robitaille É, Lefebvre M, McBrien T, Minaker LM. Rapport d'étape - Indicateurs géographiques de l'environnement alimentaire de vente au détail pour l'intervention en santé publique. Promotion de la santé et prévention des maladies chroniques au Canada. 2017;37(10):400-6.

8. Agence de la santé publique du Canada. Le fardeau économique direct des inégalités socioéconomiques en matière de santé au Canada : analyse des coûts des soins de santé selon le revenu. Ottawa (Ont.) : Agence de la santé publique du Canada; 2016. 